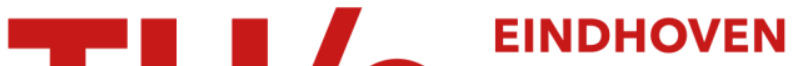

\section{Osmotic compression of droplets of hard rods: a computer simulation study}

\section{Citation for published version (APA):}

Trukhina, Y., Jungblut, S., Schoot, van der, P., \& Schilling, T. (2009). Osmotic compression of droplets of hard rods: a computer simulation study. Journal of Chemical Physics, 130(16), 164513-1/7. [164513]. https://doi.org/10.1063/1.3117924

DOI:

10.1063/1.3117924

Document status and date:

Published: 01/01/2009

\section{Document Version:}

Publisher's PDF, also known as Version of Record (includes final page, issue and volume numbers)

\section{Please check the document version of this publication:}

- A submitted manuscript is the version of the article upon submission and before peer-review. There can be important differences between the submitted version and the official published version of record. People interested in the research are advised to contact the author for the final version of the publication, or visit the $\mathrm{DOI}$ to the publisher's website.

- The final author version and the galley proof are versions of the publication after peer review.

- The final published version features the final layout of the paper including the volume, issue and page numbers.

Link to publication

\section{General rights}

Copyright and moral rights for the publications made accessible in the public portal are retained by the authors and/or other copyright owners and it is a condition of accessing publications that users recognise and abide by the legal requirements associated with these rights.

- Users may download and print one copy of any publication from the public portal for the purpose of private study or research.

- You may not further distribute the material or use it for any profit-making activity or commercial gain

- You may freely distribute the URL identifying the publication in the public portal.

If the publication is distributed under the terms of Article $25 \mathrm{fa}$ of the Dutch Copyright Act, indicated by the "Taverne" license above, please follow below link for the End User Agreement:

www.tue.nl/taverne

\section{Take down policy}

If you believe that this document breaches copyright please contact us at:

openaccess@tue.nl

providing details and we will investigate your claim. 


\title{
Osmotic compression of droplets of hard rods: A computer simulation study
}

\author{
Yu. Trukhina, ${ }^{1}$ S. Jungblut, ${ }^{2}$ P. van der Schoot, ${ }^{3}$ and T. Schilling ${ }^{1, a)}$ \\ ${ }_{1}^{1}$ Institut für Physik, Johannes Gutenberg-Universität, Staudinger Weg 7, D-55099 Mainz, Germany \\ ${ }^{2}$ Institut für Physik, Universität Wien, Boltzmanngasse 5, 1090 Wien, Austria \\ ${ }^{3}$ Faculteit Technische Natuurkunde, Technische Universiteit Eindhoven, Postbus 513, \\ 5600 MB Eindhoven, The Netherlands
}

(Received 11 February 2009; accepted 23 March 2009; published online 27 April 2009)

\begin{abstract}
By means of computer simulations, we study how droplets of hard, rodlike particles optimize their shape and internal structure under the influence of the osmotic compression caused by the presence of spherical particles that act as depletion agents. At sufficiently high osmotic pressures, the rods that make up the drops spontaneously align to turn them into uniaxial nematic liquid-crystalline droplets. The nematic droplets or "tactoids" that are formed this way are not spherical but elongated, resulting from the competition between the anisotropic surface tension and the elastic deformation of the director field. In agreement with recent theoretical predictions, we find that sufficiently small tactoids have a uniform director field, while large ones are characterized by a bipolar director field. From the shape and director-field transformation of the droplets, we are able to estimate the surface anchoring strength and an average of the elastic constants of the hard-rod nematic.
\end{abstract}

(c) 2009 American Institute of Physics. [DOI: 10.1063/1.3117924]

\section{INTRODUCTION}

Fluids of elongated particles that interact via a harshly repulsive excluded-volume interaction potential, i.e., hardrod fluids, have proven immensely useful as a model system for investigating the formation, structure, and properties of liquid crystals. ${ }^{1}$ Indeed, hard-rod fluids exhibit a wealth of aggregated states including nematic, smectic, and columnar liquid-crystalline phases as well as various plastic and crystalline solid phases, depending on the precise shape, density, and composition of the model particles present in the system. ${ }^{2}$ Closest experimental realizations of the hard-rod fluid model are found in fluid dispersions of very stiff polymers, inorganic rodlike colloids, filamentous viruses, fibrillar or tubular protein assemblies, and carbon nanotubes. ${ }^{3-7}$ For this class of system, the transition from an isotropic to a symmetry-broken liquid-crystalline or crystalline state is entropy rather than enthalpy driven, and the relevant control parameter is not the temperature but the concentration or particle density. The transitions between the different phases are driven by a tradeoff between free volume and configurational entropy.

Arguably, the experimentally and theoretically by far most extensively studied liquid-crystalline state is the nematic that is characterized by uniaxial and up-down symmetry. The preferred mean orientation of the particles is described by a director field the ground state of which is spatially uniform. The transition from the isotropic to the nematic phase is first order. (See, however, the work by Viamontes et al. $^{8}$ ) In the biphasic region it proceeds via the formation of nematic droplets called tactoids in the background isotropic dispersion. Tactoids are typically not spherical but elongated

${ }^{a)}$ Electronic mail: schillit@uni-mainz.de. and spindle shaped, and they have over the past century or so been observed in a wide variety of systems. ${ }^{9-16}$ The shape and internal structure of nematic droplets are the result of the competition between the preferred surface anchoring of the director field and the deformation of the director field that occurs if the preferred anchoring is indeed accommodated.

The shape and director-field configurations of tactoids have recently been studied theoretically in considerable detail within a macroscopic Frank elasticity theory. ${ }^{15,17-20}$ Predictions depend on several parameters: two surface energies (the surface tension and anchoring energy), three bulk elastic constants associated with the splay, twist, and bend deformations, the saddle-splay surface elastic constant, and, finally, the size of the droplet. For our purposes, it suffices to summarize the most important predictions, presuming preferential planar anchoring of the director field to the interface that for rodlike particles seems to hold for entropy reasons: (i) The director field of small drops is uniform and of large ones bipolar; (ii) the crossover is smooth and set by a healing or extrapolation length that is the ratio of an average elastic constant and an anchoring energy; (iii) the aspect ratio of uniform tactoids depends only on the anchoring energy, and of bipolar ones on their size relative to the healing length.

Some aspects of these theoretical predictions have been verified against experiment, in particular, the size dependence of aspect ratio and opening angle of the sharp ends of the tactoids. ${ }^{14,15,18-20}$ The crossover from bipolar to uniform director field has not been observed, and this probably presents quite an experimental challenge because it has been predicted to occur when the drops are quite small, that is, in the micrometer range. For such small droplets, not at all very much larger than the particles that they are made up from, director-field patterns are difficult to distinguish in polarization microscopic images. 
Clearly, computer simulations are of use here, ${ }^{21-23}$ not least because a macroscopic description might break down for such small drops, in other words, the predicted transition may be spurious. On the other hand, recent simulations on the nucleation of the nematic phase in a hard-rod fluid have indeed shown that small nuclei of the nematic phase are elongated and it appears at least that their director field is uniform. ${ }^{24}$ Simulations on small nematic droplets of prolate particles interacting via a Gay-Berne potential have been shown to exhibit similar behavior, although fluctuations are large so a director field is not so easily defined. ${ }^{25,26}$ Because systematic computational investigations of the shape and internal structure of nematic droplets are lacking, we set out to do this for nematics of hard spherocylinders of aspect ratio of 11. Our simulations build on our earlier work on hard-rod nematics in a spherical cavity of fixed size and shape, ${ }^{27}$ but we now focus on actual drops that can adjust their structure. This we do by placing the spherocylinders in a suspension of spherical particles, which interact via a hard-core repulsion with the spherocylinders yet are transparent with respect to themselves, i.e., behave like an ideal gas.

The method allows us to compress by osmotic stress droplets of a fixed number of rodlike particles. This is achieved in a controlled manner because the density of the rods in the drop will be set by the imposed pressure of the ideal gas of spheres (if we ignore a small contribution from a Laplace pressure). Hence, the drop size must then be given by the number of rods in the simulation box. Note that our making use of what in essence is a depletion agent (the spherical particles) is not all that dissimilar in spirit to the experiments of Dogic and Fraden, ${ }^{13}$ who used the nonadsorbing polymer dextran to induce phase separation in aqueous dispersions of filamentous fd virus particles.

Our results may be summarized as follows.

(i) For relatively low densities of spheres, the droplets are spherical and the rods are randomly oriented.

(ii) Above a critical sphere density, the drops become nematic and elongated.

(iii) Small tactoids have a uniform director field, and the large ones, a bipolar one.

(iv) At the crossover the drops measure about four rod lengths so indeed are very small.

(v) The surface tension anisotropy and healing length that we deduce from the simulations are consistent with theoretical estimates.

The remainder of this paper is organized as follows. In Sec. II we introduce the model and define the relevant order parameters. A detailed discussion of our results is given in Sec. III. Finally, in Sec. IV we summarize our findings and relate them to theory and experiment.

\section{MODEL AND SIMULATION DETAILS}

In the Monte Carlo simulations, we model the rods as spherocylinders consisting of a cylindrical part of length $L$ and diameter $D$, capped at both ends by hemispheres of diameter $D$. In order to stabilize droplets of rods, we surround them by a liquid of spheres. The spherocylinders interact via a hard-core repulsion. The spheres also interact with a hardcore repulsion with spherocylinders but are able to freely interpenetrate each other at no energy cost. Hence, our model is an extension of the Asakura-Oosawa (AO) model for colloid-polymer mixtures ${ }^{28,29}$ to anisotropic colloids. ${ }^{30}$ Note that strictly speaking the spheres do interact with each other via the rods while this is not so in the original AO model. This difference is of academic interest only because we consider only those concentrations of particles that produce a very strong phase separation into sphere- and rod-rich phases, so the former behaves as a (nearly) ideal gas of spheres that in essence acts as a barostat for the droplet consisting of only spherocylinders.

As already advertised in Sec. I, this implies that by means of changing the number of spheres in the system at fixed volume, we can tune the pressure exerted on the rods in the droplet. Through that we vary the density of the drop and therefore also the physical properties of the drop, such as the elastic response if the drop is in a nematic fluid state. Hence, we use the number density of spheres $\rho_{\text {sp }}$ measured far away from the droplet as a parameter that characterizes the external conditions imposed on the droplets formed. The same method has been used in an earlier study of the formation of nanocrystals in a simulation. ${ }^{31}$

The simulations were performed at fixed particle number $N$ and simulation box volume $V$ (and temperature $T$, but as the system is purely entropic, temperature is not relevant here) in a cubic box with periodic boundary conditions. We focus on spherocylinders with an aspect ratio of $L / D+1$ $=11$ (i.e., $L / D=10$ ) and spheres with a diameter twice that of the thickness of the rods, $D_{\mathrm{sp}}=2 D$. Spheres that are much larger than this introduce strong effects on the surface anchoring, while for smaller spheres the numbers needed in the simulation are so large to be unpractical from a computational point of view.

The number of spherocylinders in the box was varied from 200 to 700, and the number of spheres was fixed such that phase separation was induced into two phases containing virtually only spherocylinders or spheres. This corresponded in our simulations to between approximately 20000 and 70000 spheres. We chose the simulation box to be $70^{3} \mathrm{D}^{3}$, i.e., sufficiently large to ensure that the spherocylinders did not interact directly with each other via the periodic boundaries. We verified the droplets that form are not system spanning. The systems were equilibrated by local translation and rotation moves. Depending on the specific concentrations, $10^{6}-10^{7}$ Monte Carlo sweeps were required.

The boundary and volume of a droplet are established as follows: We first divide the system into small boxes and next verify whether a box contains the center of a sphere or whether it is intersected by any spherocylinder. In the latter case, this box is counted as a part of the droplet. If it so happens that a box does not fall into either of the two categories, it is counted as a part of the droplet if it has a larger number of nearest neighboring boxes containing spherocylinders than spheres.

As is customary, we define the average alignment of the $(N)$ rods in terms of the traceless tensor $\mathbf{Q}$, with the elements 


$$
Q_{\alpha \beta}=\frac{1}{2 N} \sum_{i=1}^{N}\left(3 u_{i \alpha} u_{i \beta}-\delta_{\alpha \beta}\right),
$$

where $u_{i \alpha}$ is the $\alpha$ component $(\alpha=x, y, z)$ of the unit vector along the axis of particle $i=1, \ldots, N$ and $\delta_{\alpha \beta}$ the Kronecker delta. Diagonalization of the tensor yields three eigenvalues, $\lambda_{+}, \lambda_{0}$, and $\lambda_{-}$, where $\lambda_{+}>\lambda_{0}>\lambda_{-}$. Different authors use different combinations of these eigenvalues to define the nematic and biaxial order parameters. ${ }^{32,33}$ To avoid confusion, we shall present all eigenvalues instead. The case $\lambda_{+}$ $>0, \lambda_{0}=\lambda_{-}$corresponds to a structure with one preferred direction. The case $\lambda_{+}=\lambda_{0}>0$ corresponds to a structure in which one direction is avoided and the two other directions are equally favored. All the intermediate cases $\lambda_{+}>0, \lambda_{+}$ $>\lambda_{0}>\lambda_{-}$correspond to a biaxial structure. Obviously, in an isotropic phase one finds $\lambda_{+}=\lambda_{0}=\lambda_{-}=0$.

When calculating observables, such as the components of the order parameter tensor properly averaged over the ensemble of configurations, all the possible types of symmetry in the system have to be taken into account. In the case of radial symmetry, we calculated the orientational tensor directly by averaging over all configurations obtained in the simulations.

If there is a preferred axis in the system along which the particles tend to align, we need to proceed differently. As rotations of the director do not cost any (free) energy, the orientation of this axis can fluctuate strongly during a simulation run. In order to average the local properties of interest, we set the reference frame for each configuration such that its director is aligned with the $z$-axis. (Note that this does not imply that we rotate the system during the simulation, we just choose a coordinate system for data accumulation.) This procedure introduces a small systematic error to the computation of the orientational order parameters, which is negligible in the context of the analysis presented in Sec. III.

\section{RESULTS AND DISCUSSION}

In the diagram of Fig. 1 we have indicated with asterisks the system conditions for which we ran the simulations. The letters "a" through "l" are used in the following to refer to specific points in this diagram. We have indicated schematically in the figure the shape and the structure of the droplets, which we discuss in more detail below. The numbers in the boxes indicate the aspect ratios of the droplets.

Figures 2 and 3 give the snapshots of two typical structures given by conditions "i" and "c", respectively, where only the spherocylinders are shown for clarity. Two essential differences between these structures can be seen: The first of the two droplets shown is (i) more elongated and (ii) the spherocylinders in this droplet have a much stronger tendency to orient in one direction. Below we will analyze these effects in a more quantitative fashion.

The difference is caused by the difference in bulk sphere density and hence osmotic stress imposed on the drop by the sphere fluid. Droplet "i" is subject to much higher pressure and hence is condensed much more than droplet "c" and has crossed over to the nematic phase. The rod densities $\rho D^{3}$ in the two drops are 0.034 and 0.027 (averaged over each drop-

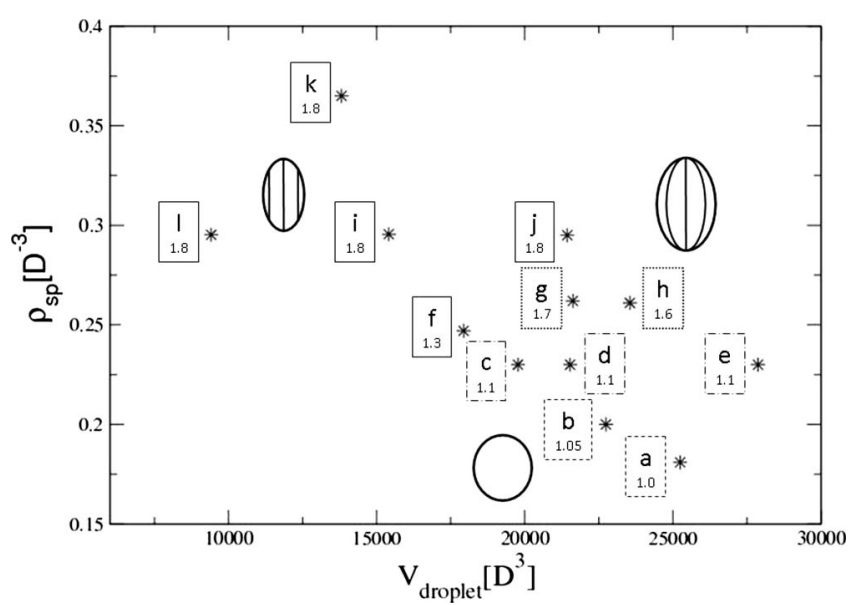

FIG. 1. "Shape" diagram of hard spherocylinder droplets immersed in a fluid of spherical particles. The horizontal axis shows the volume of the droplet of spherocylinders in units of cylinder thickness cubed, $D^{3}$, and the vertical axis the number density of the spheres far away from the droplet. The letters "a" to "l" are used in the main text to refer to specific points on the diagram. The sketches show schematically the shape and the internal structure of the droplets and distinguish spherical isotropic droplets, elongated nematic droplets with either a homogeneous, or a bipolar director field. The numbers in the boxes indicate the aspect ratios of the droplets. The boundaries of the boxes distinguish between isotropic (dashed), transition region (dash dotted), bipolar (dotted), and homogeneous (solid).

let). From the (remarkably accurate) Lee-Parsons theory of the nematic transition in bulk fluids of hard rods, ${ }^{34}$ we expect for spherocylinders of aspect ratio of 11 the nematic transition to occur at a pressure of $P D^{3} / k_{B} T \approx 0.25$, corresponding to an ideal gas density of $\rho_{\mathrm{sp}} D^{3} \approx 0.25$. This is in reasonable agreement with the transitional regime around $\rho_{\mathrm{sp}} D^{3} \approx 0.23$ in the diagram. We also estimated the transition density by simulating a compression curve and an expansion curve in the bulk and obtained $\rho_{\mathrm{sp}} D^{3} \approx 0.22$, which again is in good agreement with the numbers above. We note that due to the effects of Laplace pressure, the nematic transition should occur at a somewhat higher sphere density than that in bulk solution. For details we refer to Appendix.

In Fig. 4 we have plotted a typical isodensity distribution in the $r$ - $z$-plane for nematic droplet "i". Inside the droplet the spherocylinders have an approximately constant rod density equal to $\rho D^{3}=0.039$, which rapidly decays when approaching the interface to the fluid of spheres. It is clear that the drop is not spherical, the aspect ratio being approximately

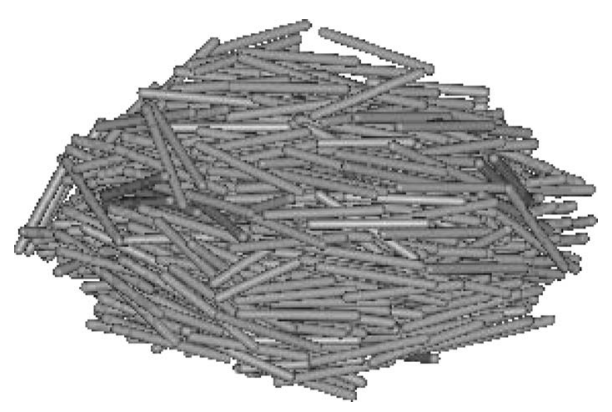

FIG. 2. A snapshot of a nematic droplet of rods that forms for the conditions indicated by "i" in Fig. 1. The spherical particles are not shown for clarity. The nematic total director is along the main axis of the drop. The average rod density in the drop is equal to $\rho D^{3}=0.034$. 


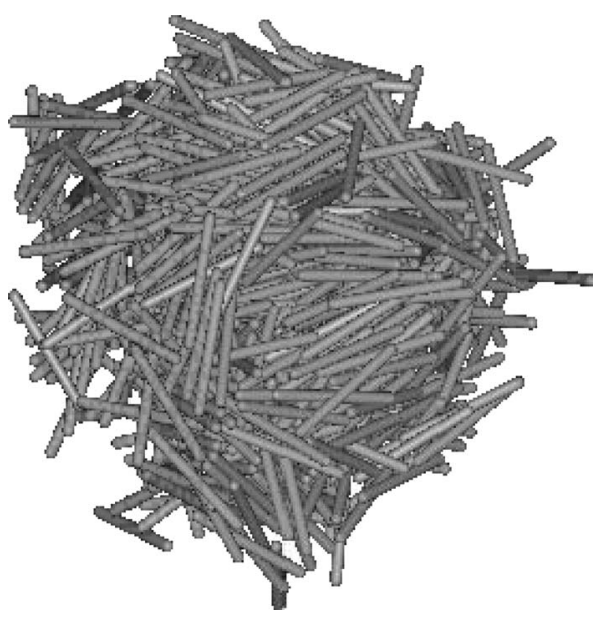

FIG. 3. A snapshot of an almost isotropic droplet of rods for conditions "c". The average rod density in the drop is equal to $\rho D^{3}=0.027$.

1.8. For comparison, and in order to determine the shape of the droplets, we cut a slice from the $r-z$-density profile at half of its maximum value. That is, where we expect the Gibbs surface to be situated. The curves obtained in this way are shown in Fig. 5 for several systems consisting of the same amount or spherocylinders $(N=500)$ but with various densities of spheres, corresponding to the points "a", "b", "c", "f", "i", and "k".

At low pressures the droplets are spherical on average. This is to be expected if the rods are in their isotropic state. However, if the pressure is increased, the droplets crossover to the nematic phase and hence become elongated in order to reduce either the elastic deformation of the director field if the anchoring is strong or the anchoring free energy if the anchoring is weak. As already advertised, the crossover occurs when the typical drop dimension exceeds the healing length, which we are going to discuss in more depth in the following section.

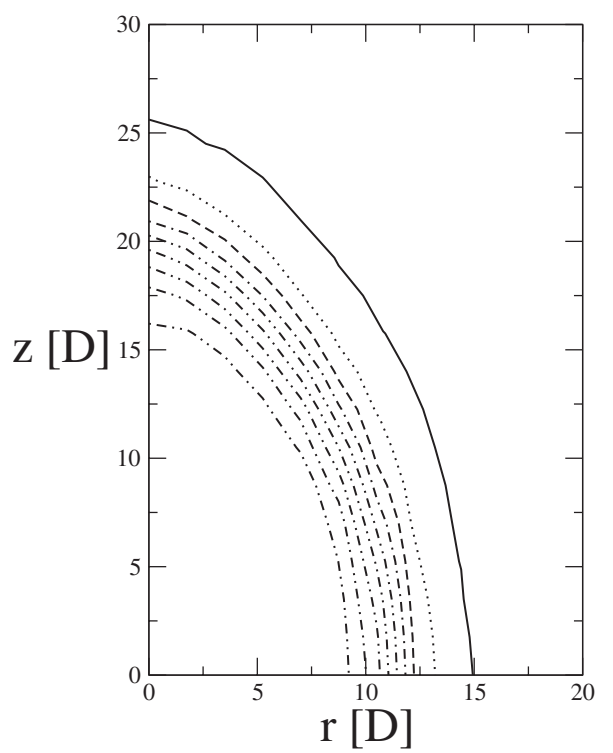

FIG. 4. Isodensity lines of spherocylinders in a droplet from conditions " $i$ ", shown in cylindrical coordinates. The solid line marks the boundary of the drop. Successive dashed, dash-dotted, etc., lines demarcate densities from $\rho D^{3}=0.039$ to $\rho D^{3}=0$.

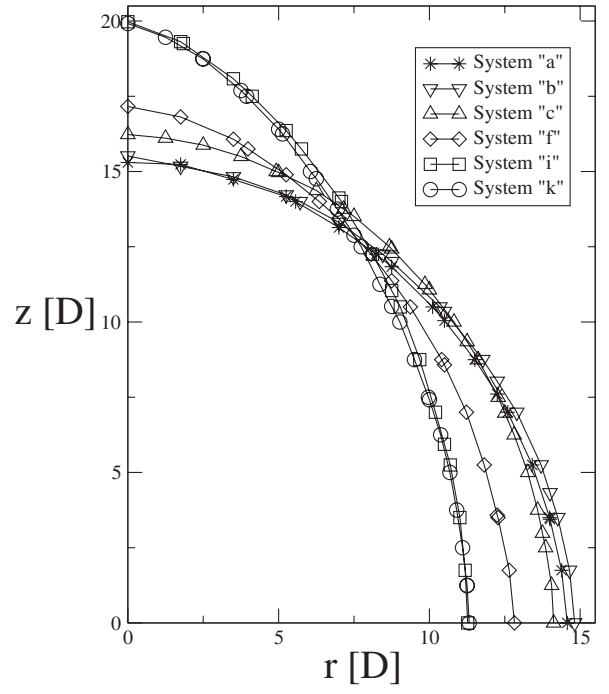

FIG. 5. The shapes of droplets consisting of 500 spherocylinders at different pressures of spheres (conditions "a", "b", "c", "f", "i", and "k" indicated in the schematic of Fig. 1). Droplets "a" and "b" are those of isotropically oriented rods, and hence more or less spherical. Drop "c" is in the transition zone from the isotropic to the nematic state. Drops "f", "i", and "k" are nematic drops, with "i" and " $k$ " exhibiting more or less uniform director fields and "f" a more bipolar one.

On increase in the density of rods, the elastic constants in all likelihood increase, too, as should the interfacial tension and potentially also the anchoring strength. We expect from scaling arguments that the ratio of the anchoring strength and the surface tension is a weak function of the pressure ${ }^{35}$ however, and in the weak anchoring regime, it is this ratio that dictates the aspect ratio of the drops. ${ }^{17}$ In the strong anchoring regime the aspect ratio is an increasing function of the healing length that in the equal-constant approximation is given by the ratio of the elastic constant and the surface tension. Hence, whether the droplets become more elongated then depends on how strongly these two energies depend on the pressure. Apparently, the elastic constants increase more strongly with pressure because the aspect ratio increases from about 1.1 to 1.8 with increasing sphere concentration.

We observe the same tendency for the systems of 700 spherocylinders. At low pressures the droplet is more or less spherical (state point "e", aspect ratio of 1.1), and at high pressures the droplet becomes elongated (state point "j”, aspect ratio of 1.8). We have to note, however, that droplet size also affects the aspect ratio: it decreases with increasing size. This has been observed experimentally and is predicted theoretically based on macroscopic theory. ${ }^{14,15,19}$ The aspect ratios for all the state points are shown in the boxes in the "shape" diagram in Fig. 1, with an estimated error of 0.1 . Note that the shape of the "spherical" droplets is spherical only on average. The lengths of the axes fluctuate by $20 \%$. Hence any given snapshot of such droplet is deformed considerably.

We now turn to the orientational state of the rods in the droplets. This is described in terms of the eigenvalues of the orientational tensor $\mathbf{Q}$ (see Sec. II). In Fig. 6 we show the $r$ and z-profiles of these eigenvalues for the drops "i", "f", and "c" (that each consist of 500 spherocylinders). The droplets 

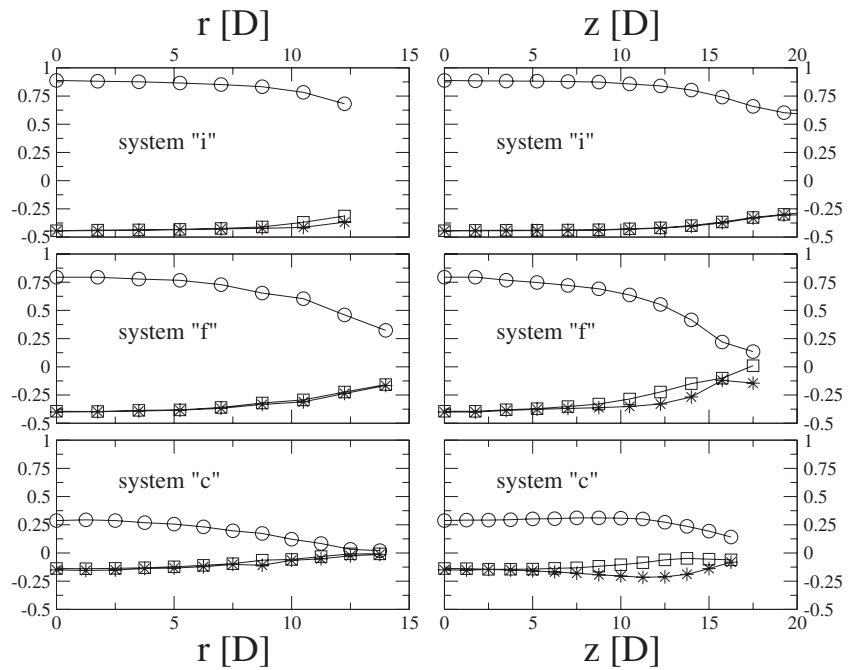

FIG. 6. The $r$ - and z-profiles of the eigenvalues of the orientational tensor $\mathbf{Q}$ for the systems "i", "f", and "c" (each consisting of 500 spherocylinders).

become less ordered and less elongated. Droplet "i" has a high nematic ordering in its center, which slightly decays on approach of the interface to the gas of spheres in both the $r$ and the $z$-direction. Droplet "f" has a lower density and also a nematic order parameter that is smaller. Droplet "c" has even lower values of the nematic order parameter, and at the interfaces all the eigenvalues become equal to zero, indicating an isotropic configuration. This drop probably is close to the conditions where the isotropic-nematic transition takes place.

By analyzing the tensor $\mathbf{Q}$ for all the investigated droplets, we construct scalar order parameter profiles as well as the nematic director-field configuration. The results are indicated schematically in the diagram in Fig. 1:

(1) Droplets "a" and "b" are spherical droplets of an isotropic rod fluid.

(2) Droplets "c"-"e" are in the isotropic-nematic transition region, exhibiting a strongly fluctuating orientational order.

(3) States "f" to "l" are strongly nematic drops.

(4) The director field of drops "i"-"l" is more or less uniform, those of " $g$ " and " $h$ " bipolar, and of "f" in between these two.

To illustrate these findings, we show the director field (the axis given by the eigenvector corresponding to the largest eigenvalue of the orientation tensor) as a function of the radial and axial distances $r$ and $z$ in Fig. 7 for the case " $k$ " and in Fig. 8 for the case " $h$ ". The director field of the tactoid $\mathrm{k}$ is more or less uniform and oriented along the main axis of the drop. That of " $h$ " is bipolar, i.e., curved along the elongated drop surface toward the tips, where the scalar order parameter drops to zero. This signifies the melting of the nematic near the tips, where theoretically one would expect the surface point defects ("boojums") to reside. ${ }^{17}$

The director field in a nematic droplet is determined by the interplay of surface anchoring and elastic forces. The bipolar structure can only be formed if the energy for bending is small enough compared to the surface energy. This can

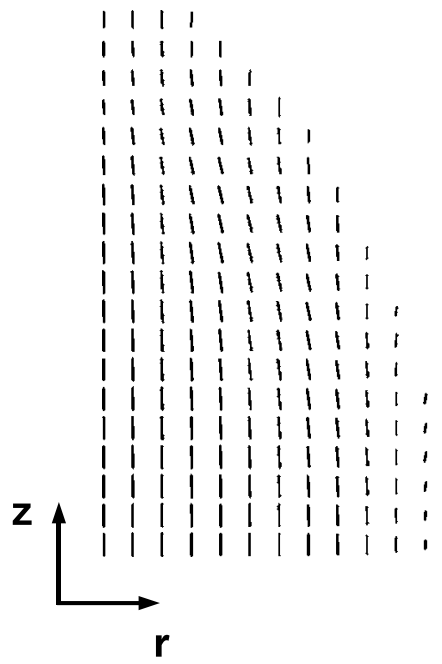

FIG. 7. An example of a nematic droplet with a homogeneous director field (system " $k$ "), the eigenvectors corresponding to the maximum eigenvalues of the orientational tensor $\mathbf{Q}$ are shown in polar coordinates.

be achieved if the density of the suspension is small (but the droplet still has a nematic structure) or if the droplet is big (and, therefore, the curvature of the interface is small). Systems "g" and " $h$ " fit into this category.

The aspect ratio of the droplets does not depend on their size if the director field inside the droplets is homogeneous. This follows directly from the well-known Wulff construction of the droplet shape given any polar angle-dependent surface tension. ${ }^{17}$ Indeed, all the droplets with an approximately homogeneous director field, droplets “i”, “j”, "k”, and "l", that we obtained in our simulations, have the same aspect ratio of 1.8 within a statistical error of the simulation of about 0.1 , in agreement with this theoretical prediction. From the aspect ratio observed in the simulations, we can, in fact, deduce a dimensionless anchoring strength. Let us presume that the anisotropic surface tension $\gamma$ has a functional form of the Rapini-Papoular type, so $\gamma=\tau\left[1+\omega(\mathbf{q} \cdot \mathbf{n})^{2}\right]$, with $\tau$ the bare surface tension, $\omega$ the dimensionless anchoring strength, $\mathbf{q}$ the surface normal, and $\mathbf{n}$ the director field at the surface of the drop. For planar anchoring to be favored, $\omega>0$. From the Wulff construction we then deduce that the aspect ratio of the

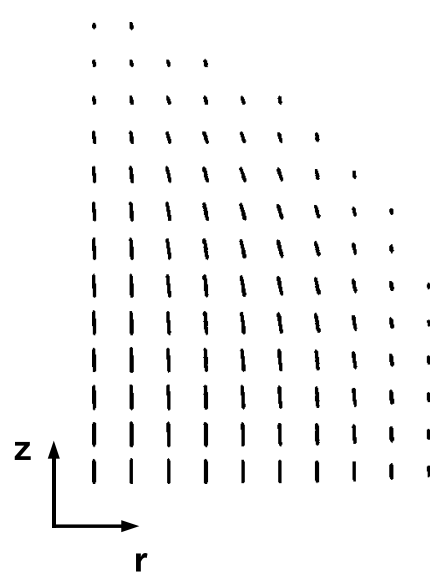

FIG. 8. An example of a nematic droplet with a bipolar director field (system " $h$ "); the eigenvectors corresponding to the maximum eigenvalues of the orientational tensor $\mathbf{Q}$ are shown in polar coordinates. 
drop equals $1+\omega$ for $0 \leq \omega \leq 1$ and $2 \sqrt{\omega}$ for $\omega>1 .{ }^{17}$ Hence, we find from our simulations a value for $\omega$ of 0.8 , quite close the value of 0.65 found by Cuetos et al. ${ }^{36}$ in a simulation study of nematic drops nucleated in a supersaturated dispersion of hard rods.

Finally, from the crossover from bipolar to homogeneous director fields, we obtain an estimate for the healing length. According to Fig. 1, the crossover occurs for drops of a volume about $1.7 \times 10^{4} D^{3}$. This value may actually depend on the sphere density, but because of the lack of any detailed information, we shall ignore this for simplicity. Presuming that the bend elastic constant is about ten times larger than the splay elastic constant, which seems reasonable on account of predictions for hard rods in the Onsager limit, the crossover occurs at a droplet volume equal to about ten times the healing length $\lambda \equiv\left(K_{11}-K_{24}\right) / \tau \omega$ cubed according to macroscopic theory, ${ }^{18,19}$ where $K_{11}$ denotes the splay elastic constant and $K_{24}$ the saddle-splay surface elastic constant. So, we find for the healing length $\lambda \approx 12 D$, which is about a rod length. Clearly, macroscopic theories, such as those of Kaznacheev et al. ${ }^{15,20}$ and of Prinsen and van der Schoot, ${ }^{17-19}$ could perhaps be expected to break down at such small length scales, yet the predicted crossover from uniform to bipolar director fields apparently still survives.

From the estimate of the healing length, we can obtain an order of magnitude estimate of the interfacial tension between the rods and the spheres that we can compare with the scaling estimate given in Appendix. If we presume the Saupe-Nehring relation to hold, implying that $K_{24}=\left(K_{11}\right.$ $\left.-K_{22}\right) / 2{ }^{37}$ and make use of the approximate expression $K_{22}=K_{11} / 3,{ }^{38}$ we obtain $\lambda \approx 2 K_{11} / 3 \tau \omega$. Hence, $K_{11} / \tau$ $\approx 14 D$ or $\beta \tau D^{2} \approx \beta K_{11} D / 14$, where $\beta$ is defined as the inverse thermal energy $1 / k_{B} T$ with $k_{B}$ the Boltzmann's constant and $T$ the absolute temperature. Within a second-virial approximation, which admittedly is not very accurate for rods of aspect ratio below 20 , we expect $\beta K_{11} D \approx 0.9$ to hold near the transition. ${ }^{39}$ Hence, for the interfacial tension between the rods and the spheres, we obtain the estimate $\beta \tau D^{2} \approx 0.07$. According to our scaling estimate cited in Appendix, we have $\beta \tau D^{2} \approx 0.25 \alpha \xi / D$ with $\alpha$ a prefactor that should be of order of 0.1 (Ref. 35) and $\xi$ the interfacial width. If $\xi \approx L$, this then implies that $\alpha \approx 0.03$, which is somewhat smaller than expected. ${ }^{35}$

In order to go beyond this qualitative analysis, simulation data of the elastic constants of the bulk nematic and of the surface tension between the coexisting bulk fluids would be necessary. Unfortunately, these are not available yet for spherocylinders of aspect ratio of 11, as, in particular, simulations to determine elastic constants are computationally rather expensive. (To our knowledge, elastic constants have been computed only for spherocylinders of aspect ratio of $\left.6 .^{40}\right)$

\section{SUMMARY}

By means of computer simulations we have shown that fluid droplets of hard rods, osmotically compressed by the presence of spherical particles, undergo an isotropic-nematic transition at sufficiently high osmotic stress. We find the nematic droplets not to be spherical but elongated. The director field of the drops is uniform if smaller than a critical size and bipolar if larger than that. We interpret our findings in terms of the predictions of continuum mechanical theory that minimizes the combined effect of an elastic deformation of the director field and an anchoring frustration of this director field at the surface of the drops. Although in our simulations the drops are not at all large on the scale of the rods, and continuum theory should perhaps not be expected to be accurate, results from both levels of description seem to be consistent with each other down to drop sizes that are as small as a few times the particle length.

\section{ACKNOWLEDGMENTS}

We thank K. Binder and M. Allen for helpful suggestions. CPU time was provided on the JUMP by the John von Neumann Centre in Jülich. We thank the Deutsche Forschungsgemeinschaft (DFG, Emmy Noether Program No. Schi853/1, and SFB Tr6, Project D5) and the MWFZ Mainz for financial support. Moreover, we thank an anonymous referee for spotting an error of sign.

\section{APPENDIX: ESTIMATE OF THE ROD DENSITY IN THE DROP}

An estimate for the rod density in the droplet as a function of the number $N$ of rods and the density of spheres $\rho_{\mathrm{sp}}$ can be obtained by presuming complete demixing of the two components and by presuming that the interface between them is sharp. Let $R$ be the radius of the drop, assumed perfectly spherical, then $\rho=3 N / 4 \pi R^{3}$ is the density of the rods in drop. The bulk pressure of the hard rods is to a very good approximation equal to the expression put forward by Parsons and by Lee, ${ }^{7}$

$$
\beta P=\rho\left(1+\frac{2 \phi(2-\phi)}{(1-\phi)^{3}}\left[1+\frac{3 \pi}{8} \Lambda\right]\right),
$$

at least in the isotropic phase, where $\phi=\rho\left(\pi D^{3} / 6\right.$ $\left.+\pi L D^{2} / 4\right)$ denotes the packing fraction, $\Lambda=(L / D)^{2} / \pi(1$ $+3 L / 2 D$ ) for slender rods is proportional to their aspect ratio, and $\beta$ denotes the reciprocal thermal energy $1 / k_{B} T$ with $k_{B}$ Boltzmann's constant and $T$ the absolute temperature. The pressure of the ideal gas of spheres obeys

$$
\beta P_{\mathrm{sp}}=\rho_{\mathrm{sp}} .
$$

Mechanical equilibrium between the gas of hard rods and that of ideal spheres demands that

$$
P-\frac{2 \gamma}{R}=P_{\mathrm{sp}},
$$

where the second term on the left-hand side is the contribution from the Laplace pressure across the curved interface, with $\gamma$ the interfacial tension that presumably depends on the bulk densities of both the rods and the spheres. For any given number of rods $N$, this equation sets the equilibrium size of the drop.

An estimate of the magnitude of the Laplace pressure may be given by making use of the scaling ansatz $\gamma \approx P_{\mathrm{sp}} \xi$, with $\xi \approx L$ the actual interfacial width. ${ }^{35}$ Hence, $P / P_{\mathrm{sp}} \approx 1$ 
$+2 \alpha \xi / R$ with $\alpha$ a constant of proportionality that we estimate to be of order of $0.1 .^{35}$ So, the presence of the interface increases the pressure in the drop relative to that in the reservoir of spheres and hence induces the onset of the nematic phase at lower densities of spheres the smaller the drop.

From Table I of Lee, ${ }^{41}$ we deduce by linear interpolation that for rods of $L / D=10$, the bulk nematic phase sets in at a dimensionless pressure $\beta P D^{3} \approx 0.247$, corresponding to a sphere fraction of $\rho_{\mathrm{sp}} D^{3} \approx 0.247$, in reasonable agreement with what we find in the simulations. (See Fig. 1.)

${ }^{1}$ P. G. de Gennes and J. Prost, The Physics of Liquid Crystals (Clarendon, Oxford, 1993).

${ }^{2}$ P. Bolhuis and D. Frenkel, J. Chem. Phys. 106, 666 (1997).

${ }^{3}$ Z. Dogic and S. Fraden, Curr. Opin. Colloid Interface Sci. 11, 47 (2006).

${ }^{4}$ P. Davidson and J. C. P. Gabriel, Curr. Opin. Colloid Interface Sci. 9, 377 (2005).

${ }^{5}$ J. Kas, H. Strey, J. X. Tang, D. Finger, R. Ezzell, and E. Sackmann, Biophys. J. 70, 609 (1996).

${ }^{6}$ S. J. Zhang and S. Kumar, Small 4, 1270 (2008).

${ }^{7}$ G. J. Vroege and H. Lekkerkerker, Rep. Prog. Phys. 55, 1241 (1992).

${ }^{8}$ J. Viamontes, P. W. Oakes, and J. X. Tang, Phys. Rev. Lett. 97, 118103 (2006).

${ }^{9}$ A. S. Sonin, Colloid J. USSR 60, 129 (1998).

${ }^{10}$ J. D. Bernal and I. Fankuchen, Nature (London) 139, 923 (1937).

${ }^{11}$ J. D. Bernal and I. Frankuchen, J. Gen. Physiol. 25, 111 (1941).

${ }^{12}$ H. Zocher and C. Toeroek, Kolloid-Z. 170, 140 (1960).

${ }^{13}$ Z. Dogic and S. Fraden, Philos. Trans. R. Soc. London, Ser. A 359, 997 (2001).

${ }^{14}$ P. W. Oakes, J. Viamontes, and J. X. Tang, Phys. Rev. E 75, 061902 (2007).

${ }^{15}$ A. V. Kaznacheev, M. M. Bogdanov, and A. S. Sonin, J. Exp. Theor Phys. 97, 1159 (2003).

${ }^{16}$ M. C. D. Mourad, E. J. Devid, M. M. van Schooneveld, C. Vonk, and H.
N. W. Lekkerkerker, J. Phys. Chem. B 112, 10142 (2008).

${ }^{17}$ P. Prinsen and P. van der Schoot, Phys. Rev. E 68, 021701 (2003).

${ }^{18}$ P. Prinsen and P. van der Schoot, Eur. Phys. J. E 13, 35 (2004).

${ }^{19}$ P. Prinsen and P. van der Schoot, J. Phys.: Condens. Matter 16, 8835 (2004).

${ }^{20}$ A. V. Kaznacheev, M. M. Bogdanov, and S. A. Taraskin, J. Exp. Theor. Phys. 95, 57 (2002).

${ }^{21}$ A. Golemme, S. Zumer, D. W. Allender, and J. W. Doane, Phys. Rev. Lett. 61, 2937 (1988).

${ }^{22}$ J. H. Erdmann, S. Zumer, and J. W. Doane, Phys. Rev. Lett. 64, 1907 (1990).

${ }^{23}$ O. O. Prishchepa, A. V. Shabanov, and V. Y. Zyryanov, Phys. Rev. E 72, 031712 (2005).

${ }^{24}$ A. Cuetos and M. Dijkstra, Phys. Rev. Lett. 98, 095701 (2007).

${ }^{25}$ R. Berardi, A. Costantini, L. Muccioli, S. Orlandi, and C. Zannoni, J. Chem. Phys. 126, 044905 (2007).

${ }^{26}$ M. A. Bates, Chem. Phys. Lett. 368, 87 (2003).

${ }^{27}$ Y. Trukhina and T. Schilling, Phys. Rev. E 77, 011701 (2008).

${ }^{28}$ F. Oosawa and S. Asakura, J. Chem. Phys. 22, 1255 (1954).

${ }^{29}$ A. Vrij, Pure Appl. Chem. 48, 471 (1976).

${ }^{30}$ S. Jungblut, R. Tuinier, K. Binder, and T. Schilling, J. Chem. Phys. 127, 244909 (2007).

${ }^{31}$ M. Grünwald, C. Dellago, and P. L. Geissler, J. Chem. Phys. 127, 154718 (2007).

${ }^{32}$ R. Eppenga and D. Frenkel, Mol. Phys. 52, 1303 (1984).

${ }^{33}$ R. J. Low, Eur. J. Phys. 23, 111 (2002).

${ }^{34}$ A. Cuetos, B. Martinez-Haya, and S. Lago, Phys. Rev. E 75, 061701 (2007).

${ }^{35}$ P. van der Schoot, J. Phys. Chem. B 103, 8804 (1999).

${ }^{36}$ A. Cuetos, R. van Roij, and M. Dijkstra, Soft Matter 4, 757 (2008).

${ }^{37}$ J. Nehring and A. Saupe, J. Chem. Phys. 54, 337 (1971).

${ }^{38}$ S.-D. Lee and R. B. Meyer, J. Chem. Phys. 84, 3443 (1986).

${ }^{39}$ G. J. Vroege and T. Odijk, J. Chem. Phys. 87, 4223 (1987).

${ }^{40}$ B. Tjipto-Margo, G. T. Evans, M. P. Allen, and D. Frenkel, J. Phys. Chem. 96, 3942 (1992).

${ }^{41}$ S. Lee, J. Chem. Phys. 87, 4972 (1987). 